\title{
SELF-UNLINKED SIMPLE CLOSED CURVES
}

\author{
BY \\ DAVID W. HENDERSON(1)
}

1. Discussion of results. This paper is a sequel to [4] and all the definitions and notations of [4] will be assumed. In addition, the numbering of the theorems in the present paper has been made to follow the numbering of [4].

A simple closed curve $J$ in a space $M$ is said to be self-unlinked, if there exist a mapping $h: J \times[0,1] \rightarrow M$ such that

(a) $h \mid J \times\{0\}=$ inclusion of $J$ in $M$,

(b) $h(J \times\{1\})=$ a point, and

(c) $h(J \times(0,1]) \subset M-J$.

In [4] we proved, as a partial answer to Question IV.1, that (IV.2) every selfunlinked tame simple closed curve (scc) in a 3-manifold bounds a disk. In this paper we investigate this question when we allow the scc's to be wild.

First we give some pertinent definitions, for which it will be assumed that everything is in a 3-manifold $M$. A complex is wild if it is not tame (see I. 11 of [4]). A 0 -dimensional set is tame if, for every $\varepsilon>0$, it can be covered by the interiors of a collection of disjoint 3-cells each of diameter less than $\varepsilon$. A set $X$ is locally tame at $p$ if $p$ has a closed neighborhood in $X$ which is a tame complex in $M$. If $X$ is not locally tame at $p$ then $p$ is a wild point of $X$. A set is called nicely wild if the union of its wild points is a tame 0-dimensional set.

For $J$ an arc or scc we make the following definitions, the first of which is used in [1]. The penetration index $P(J, x)$ of $J$ at a point $x \in J$ is the smallest cardinal number $n$ such that there are arbitrarily small 2-spheres enclosing $x$ and containing no more than $n$ points of $J$. The penetration index $P(J)$ of $J$ is the least upper bound of the cardinal numbers $P(J, x)$, for all $x \in J$. If $J$ is nicely wild, then the nice penetration index $N P(J)$ of $J$ is the smallest integer $n$ such that, for every $\varepsilon>0$, the set of wild points of $J$ can be covered by the interiors of a collection of disjoint 3-cells each with diameter less than $\varepsilon$ and such that the boundary of each 3 -cell intersects $J$ in no more than $n$ points. (The union of members of this collection is called a taming $\varepsilon$-set of $J$ of index $n$.)

CONJECTURE. There is a nicely wild scc $J$ such that $N P(J) \neq P(J)$.

Received by the editors February 20, 1965.

(1) This paper consists of portions of the author's Ph.D. thesis written under the supervision of R. H. Bing and while the author was a National Science Foundation Graduate Fellow. The author wishes to thank the referee for his helpful suggestions. 
The author expects such an example because he knows of a nicely wild scc $J$ which has a point $x$ such that $P(J, x)=3$; and, for any $J, N P(J)$ is even.

In the definition of nice penetration index we may require that the 3-cells are tame, because of the following:

THEOREM V.1. Suppose every set of diameter less than $\varepsilon$ in $M$ lies in the interior of a convex 3-cell. (For instance, metrize $M$ with the barycentric metric and let $\varepsilon$ be less than 1.) If $J$ is a nicely wild scc that is locally polyhedral mod its wild points, and if $T$ is a taming $\varepsilon$-set of $J$ of finite index, then there is a polyhedral taming $\varepsilon$-set $T^{\prime}$ of $J$ with the same number of components as $T$, such that $\mathrm{Bd} T^{\prime} \cap J$ has no more points than $\operatorname{Bd} T \cap J$ and $J$ pierces $\operatorname{Bd} T^{\prime}$ at each point of intersection.

The principal results of this section are the following theorems.

In each $J$ is a self-unlinked, nicely wild scc in a 3-manifold $M$, and we further suppose that $J$ is locally polyhedral $\bmod W(W \equiv$ set of wild points of $J)$.

THEOREM V. 2. J bounds an s-disk $D$ which is locally polyhedral $\bmod W$, and $|J| \cap \mid$ int $D \mid=\varnothing$.

THEOREM V.3. If either

(a) $N P(J)=2$, or

(b) $N P(J)$ is finite and $J$ has only finitely many wild points,

then there is an s-disk $D^{\prime}$ and a sequence $\left\{T_{i}\right\}$ such that

(a) for each $i, T_{i}$ is a taming $\frac{1}{2} i$-set of $A$ of index $N P(J)$,

(b) $\left|\operatorname{Bd} D^{\prime}\right|=J$ and $\left[D^{\prime} \mid D^{\prime-1}\left(\left|D^{\prime}\right|-W\right)\right.$, Bd $\left.D^{\prime} \mid D^{\prime-1}(J-W)\right]$ is in rnp in $M-W$, and

(c) for each $i$, there is an $s$-disk $D_{i}$ such that

(i) $\left(D_{i}, \mathrm{Bd} D_{i}\right)$ is in rnp in $\left(M-\operatorname{int} T_{i}, J+\mathrm{Bd} T_{i}\right)\left({ }^{2}\right)$,

(ii) $\left|D_{i}\right| \supset\left|D_{i-1}\right|$, and

(iii) $D^{\prime}$ equals the limit of the $D_{i}$ 's, as maps.

THEOREM V.4. If $J$ bounds an s-disk $D^{\prime}$ satisfying the stated conclusion of Theorem V.3, then $J$ bounds a nonsingular disk $D$.

THEOREM V.5. If $N P(J) \leqq 4$ and $J$ has only finitely many wild points, then $N P(J)=2$.

An immediate consequence of V.4, V.5 and the characterization of tame scc's by O. G. Harrold, H. C. Griffith, and E. E. Posey in [3] is the following:

THEOREM V.6. If either

(a) $N P(J)=2$, or

(b) $N P(J) \leqq 4$ and $J$ has only finitely many wild points, then $J$ is tame.

(2) Bd $T_{i}+J$ is not a 2-manifold, but everything makes sense since $S\left(D_{i}\right) \subset \mathrm{Bd} T_{i}$. 
If $J$ is a scc on Alexander's Horned Sphere, $S$, which contains all the wild points of $S$, then

(a) $J$ is a wild, nicely wild scc,

(b) $N P(J)=P(J)=4$, and

(c) $J$ bounds a disk.

In addition, by "tying the Fox'-Artin knot with a pointed ribbon" one can obtain a scc $J$ such that

(a) $J$ is a wild, nicely wild scc with one wild point,

(b) $N P(J)=P(J)=6$, and

(c) $J$ bounds a disk.

Finally, by tying a convergent sequence of knots in a scc, one obtains a scc $J$ such that

(a) $J$ is a nicely wild scc with one wild point,

(b) $N P(J)=P(J)=2$,

(c) but $J$ is wild.

2. Proof of V.1. Let $C$ be a component of $T$. Theorem V.1 will follow if we produce a polyhedral 3-cell $B$ such that $W \cap$ int $C=W \cap$ int $B(W=$ set of wild points of $J), J$ pierces $B d B$ at each point of $J \cap B d B$, diameter of $B<\varepsilon, B d B \cap J$ has no more points than $\mathrm{Bd} C \cap J$, and $B$ does not intersect any other components of $T$.

Let $\delta$ be a positive number less than each of $(\varepsilon-($ diameter of $C)),(1 / 3)$ (distance from $C$ to $T-C$ ), and $(1 / 3)$ (distance from $\mathrm{Bd} C$ to $W$ ). By the approximation theorems of [2] we may assume that $\mathrm{Bd} C$ is locally polyhedral mod $J \cap \mathrm{Bd} C$.

Enclose each point $p$ of Bd $C \cap J$ by a polyhedral 2-sphere $S_{p}$ such that each $S_{p}$ is so small that

(a) the diameter of $S_{p}$ is less than $\delta$,

(b) the $S_{p}$ 's are disjoint,

(c) $S_{p} \cap J$ is two points at each of which $J$ pierces $S_{p}$,

(d) $S_{p}$ is in general position with respect to $\mathrm{Bd} C$, and

(e) there is a component $K$ of $\mathrm{Bd} C-\sum S_{p}$ which separates the $\left(\mathrm{Bd} C \cap S_{p}\right)$ 's on $\mathrm{Bd} C$.

$\operatorname{cl}(K)(\mathrm{cl}=$ closure $)$ is a disk with holes and each component of $\operatorname{Bd}(\operatorname{cl}(K))$ is a scc on some $S_{p}$. For each $p$, only one scc of $\operatorname{cl}(K) \cap S_{p}$ bounds a disk in $\mathrm{Bd} C-K$ that intersects $J$. Therefore, since by hypothesis $C+\sum S_{p}$ is contained in the interior of a 3-cell, we may use linking arguments in $E^{3}$ to show that, for each $p$, all components but one of $\operatorname{cl}(K) \cap S_{p}$ bounds a disk on $S_{p}-J$, and that the other one bounds a polyhedral disk on $S_{p}$ that intersects $J$ at most once. We can make these disks disjoint by pushing their interiors slightly to one side. Then $K$ plus the above disks is a polyhedral 2-sphere $S$ in a convex 3-cell of $M$. Let $B$ be the 3-cell bounded by $S$.

Clearly $\mathrm{Bd} B \cap J$ has no more points than $\mathrm{Bd} C \cap J$, diameter of $B<\varepsilon$, and $B$ does not intersect any other components of $T$. Let $w \in W \cap C$ and let $\lambda$ be a general 
position arc from $w$ to $M-\left(C+\sum S_{p}\right)$ which misses the $S_{p}$ 's. [This arc is possible since the 3-cells bounded by $S_{p}$ have diameter less than (1/3) (distance from $\operatorname{Bd} C$ to $W)$.] Then $\lambda \cap \operatorname{Bd} B=\lambda \cap K=\lambda \cap \operatorname{Bd} C$; thus, since $B+C$ is in the interior of a 3-cell and $w \in C, \lambda \cap \mathrm{Bd} B$ is an odd number of points and, therefore, $w \in B . B$ is the desired 3-cell.

3. Proof of V.2. The proof of V.2 parallels the proof of III.4 of [4] and thus will only be sketched here.

$|J|-\mathrm{W}$ is an infinite 1-dimensional polyhedral graph in $M$. Since $J$ is selfunlinked we may assume that $J$ is the boundary of an $s$-disk $D$ and that $D$ is polyhedral mod $J$. Consider $|J|-W$ as a subcomplex of some subdivision $\alpha$ of $M-W$.

Let $\Delta$ be the standard disk and let $\Omega \equiv D^{-1}(W)$.

Now go through the proof of III.4 replacing $M$ by $M-W,|L|$ by $|J|-W, D$ by $D \mid(\Delta-\Omega), \Delta$ by $\Delta-\Omega$, et cetera. Choose $\Delta^{\prime}$ so that $\operatorname{Bd} \Delta^{\prime} \cap \operatorname{Bd} \Delta=\Omega$.

4. Proof of V.3. We shall assume that $M$ is so metrized that every set of diameter no more than 1 lies in a convex 3-cell (for example, the barycentric metric).

Let $D$ be the disk promised by V.2 and (using II.2 of [4] in $M-W$ ) suppose that $\left(D\left|D^{-1}(|D|-W), \operatorname{Bd} D\right| D^{-1}(J-W)\right)$ is in $\operatorname{rnp}$ in $M-W$.

Let $\varepsilon_{1}=1$, if $N P(J)=2$; otherwise let $\varepsilon_{1}$ be a positive number less than 1 and so small that, if $k$ is the number of points in $W$, then there is a positive integer $n \leqq N P(J) \times k$ such that

(4.1) no taming $\varepsilon_{1}$-set $T$ of $J$ of index $\leqq N P(J)$ has fewer than $k$ components nor does $B d T \cap J$ have fewer than $n$ points.

Let $\delta(\varepsilon)$ be a positive number less than $\varepsilon / 3$ so small that

(4.2) if $\Delta^{\prime}$ is a subdisk of $\Delta$ (the standard disk) and $\operatorname{diam}\left(D\left(\operatorname{Bd} \Delta^{\prime}\right)\right)<\delta(\varepsilon)$, then $\operatorname{diam} D\left(\Delta^{\prime}\right)<\varepsilon / 3$.

Let $\Delta_{1}, \Delta_{2}, \cdots, \Delta_{i}, \cdots$ be an expanding sequence of proper subdisks of int $\Delta$ so that $\left\{\mathrm{Bd} \Delta_{i}\right\}$ converges uniformly to $\operatorname{Bd} \Delta$.

Choose $T_{1}$ so that

(4.3) $T_{1}$ is a polyhedral (see VI.1) taming $\delta\left(\varepsilon_{1}\right)$-set of $J$ of index $N P(J)$,

(4.4) all components of $T_{1}$ intersect $W$,

(4.5) if $N P(J) \neq 2, T_{1}$ has only $k$ components and $\mathrm{Bd} T_{1} \cap J$ has $n$ points (see (4.1)), and

(4.6) $T_{1} \subset M-D\left(\Delta_{1}\right)$.

We may suppose that $\mathrm{Bd} T_{1}$ and $D$ are in general position so that

$$
D^{-1}\left(B d T_{1} \cap|D|\right)
$$

is a finite collection of disjoint scc's and spanning arcs of $\Delta$ in $\Delta-\Delta_{1}$.

Let $K$ be the component of $\Delta-D^{-1}\left(\operatorname{Bd} T_{1} \cap|D|\right)$ containing $\Delta_{1}$. The boundary of $K$ is a finite collection of scc's in $D^{-1}\left(\operatorname{Bd} T_{1} \cap|D|\right)+\operatorname{Bd} \Delta$. Let $E_{1}$ be the smallest disk in $\Delta$ containing $K$. (Note that $\operatorname{Bd} K \cap \operatorname{Bd} \Delta \subset \operatorname{Bd} E_{1} \subset \operatorname{Bd} K$.) 
If $A$ is a member of $\mathfrak{A}$ (those scc's of $D^{-1}\left(\mathrm{Bd} T_{1}\right) \cap \operatorname{int} E_{1}$ which can be shrunk to a point in $\left.\mathrm{Bd} T_{1}-J\right)$ and $E_{a}$ is the disk that $A$ bounds in $E_{1}$, then we can replace $D\left(E_{a}\right)$ by the singular disk which $D(A)$ bounds on $\mathrm{Bd} T_{1}-J$. By pushing this disk slightly to one side of $\mathrm{Bd} T_{1}$ we can remove a component of $D^{-1}\left(\operatorname{Bd} T_{1} \cap|D|\right)$. If we apply the above "disk-switching and pushing" only to outermost (in $E_{1}$ ) members of $\mathfrak{U}$ then no point of $\Delta$ will have its image changed more than once.

Thus, by applying the "disk-switching and pushing" to each outermost (in $E$ ) member of $\mathfrak{U}$ and then II.2 of [4] we obtain an $s$-disk $D_{1}^{\prime}$ such that

(4.7) $D_{1}^{\prime}\left|\Delta_{1}+\left(\Delta-\operatorname{int} E_{1}\right)+\operatorname{Bd} \Delta=D\right| \Delta_{1}+\left(\Delta-\operatorname{int} E_{1}\right)+\operatorname{Bd} \Delta$,

(4.8) $D_{1}^{\prime-1}\left(\mathrm{Bd} T_{1} \cap\left|D_{1}^{\prime}\right|\right) \cap \operatorname{int} E_{1}$ is a finite collection of scc's whose images under $D_{1}^{\prime}$ cannot be shrunk to a point on $\mathrm{Bd} T_{1}-J$, and

(4.9) $\left(D_{1}^{\prime}-W, \mathrm{Bd} D_{1}^{\prime}-W\right)$ is in $\operatorname{rnp}$ in $M-W$.

Let $\mathfrak{B}$ be the collection of all components (scc's) of $D_{1}^{\prime-1}\left(B d T_{1} \cap\left|D_{1}^{\prime}\right|\right) \cap \operatorname{int} E_{1}$. If $\mathfrak{B} \neq \varnothing$, let $A$ be an innermost (in $E_{1}$ ) scc of $\mathfrak{B}$. $A$ bounds a disk $E_{a} \subset E_{1}$ and $D_{1}^{\prime}\left(E_{a}\right) \subset\left|D_{1}^{\prime}\right|-\operatorname{int} T_{1}$, or $T_{1}-J$. We shall treat these two cases separately.

If $D_{1}^{\prime}\left(E_{a}\right) \subset T_{1}-J$, then, since $D_{1}^{\prime}(A)$ cannot be shrunk on $\mathrm{Bd} T_{1}-J$, we can use the loop theorem to get a scc $J_{a}$ such that $J_{a}$ bounds a disk $D_{a}$ in $T_{1}-J$ but each of the two disks which $J_{a}$ bounds on Bd $T_{1}$ contain points of $J \cap \mathrm{Bd} T_{1}$. Thus $D_{a}$ separates $J \cap C$, where $C$ is the component of $T_{1}$ containing $J_{a}$. If we "cut" $C$ apart along $D_{a}$ (this cut could be accomplished by removing from $C$ the interior of a regular neighborhood of $D_{a}$ that misses $J$ ), we obtain a new taming $\delta$-set $T^{\prime}$ of $J$.

If $N P(J)=2$, then $J \cap \mathrm{Bd} C$ is two points and $J$ intersects the boundary of each part of the "cut apart" $C$ in only one point. But a scc that intersects a 2 -sphere only once is contained wholly in one complementary domain or the other; therefore, $J \cap C$ is two points and $C$ contains no points of $W$. This is a contradiction of (4.4).

If $N P(J) \neq 2$, then $T^{\prime}$ is a taming $\delta\left(\varepsilon_{1}\right)$-set of index $N P(J)$ and with $k+1$ components. But since $W$ has only $k$ points one of the components, $C^{\prime}$ say, of $T^{\prime}$ does not intersect $W$. But then $T^{\prime}-C^{\prime}$ is a taming $\delta\left(\varepsilon_{1}\right)$-set of index $N P(J)$ and with $k$ components such that $\operatorname{Bd}\left(T^{\prime}-C^{\prime}\right) \cap J$ has fewer points than $\operatorname{Bd} T \cap J$ which contradicts (4.1), (4.3), and (4.5).

Thus $D_{1}^{\prime}\left(E_{a}\right)$ is not contained in $T_{1}-J$.

If $D_{1}^{\prime}\left(E_{a}\right) \subset\left|D_{1}^{\prime}\right|-\left(\right.$ int $\left.T_{1}+J\right)$, then by the loop theorem there is a real disk $E_{a}$ such that int $E_{a}$ is contained in $M-\left(T_{1}+J\right)$. Also each of the disks $E_{a}^{\prime}$ and $E_{a}^{\prime \prime}$ which $\mathrm{Bd} E_{a}$ bounds on $\mathrm{Bd} T_{1}$ contains points of $J \cap \mathrm{Bd} T_{1}$. Because of (4.2) and (4.3), the diameter of $E_{a}$ is less than $\varepsilon_{1} / 3$. Thus one of $E_{a}+E_{a}^{\prime}$ or $E_{a}+E_{a}^{\prime \prime}$, say $E_{a}+E_{a}^{\prime}$, is a 2-sphere of diameter less than $2 \varepsilon_{1} / 3$ not containing $C$ (the component of $T_{1}$ containing $\mathrm{Bd} E_{a}$ ) in its small complementary domain. Thus $E_{a}+E_{a}^{\prime}$ lies in a convex 3-ball of $M$ (see note at beginning of $\S 4$ ) and thus bounds a 3-cell $B$ of diameter less than $2 \varepsilon_{1} / 3$. 
$C+B$ is a 3-cell and $J \cap \operatorname{Bd}(C+B)=J \cap E_{a}^{\prime \prime}$ has fewer points than $J \cap \operatorname{Bd} C$. Thus, if $N P(J)=2, J \cap \operatorname{Bd}(C+B)$ is one point and $C \subset C+B$ does not intersect $W$, which contradicts (4.4). If $N P(J) \neq 2$, then $\operatorname{Bd}\left(T_{1}+B\right) \cap J$ has fewer points than $\mathrm{Bd} T_{1} \cap J$ which contradicts (4.1), (4.2), (4.3), and (4.5).

Thus we conclude that $\mathfrak{B}$ is empty and that $D_{1}=D_{1}^{\prime} \mid E_{1}$ is an $s$-disk satisfying (c) (i) of V.3, if $D_{1}^{\prime}$ is substituted for $D^{\prime}$. With the same substitution $T_{1}$ and $D_{1}^{\prime}$ satisfy (a) and (b) of V.3.

We now repeat the above process letting $\varepsilon_{2}$ be a positive number less than $\varepsilon_{1}$ and $\frac{1}{2}$ and with the following substitutions: $\varepsilon_{2}$ for $\varepsilon_{1}, D_{1}^{\prime}$ for $D, D_{2}^{\prime}$ for $D_{1}^{\prime}, E_{2}$ for $E_{1}, \Delta_{2}+E_{1}$ for $\Delta_{1}, D_{2}$ for $D_{1}$, and $T_{2}$ for $T_{1}$. We can choose $T_{2}$ to satisfy $T_{2} \subset M-D_{1}^{\prime}\left(\Delta_{2}+E_{1}\right)$ since $D_{1}^{\prime-1}(W)=D^{-1}(W) \subset \mathrm{Bd} \Delta-E_{1}$. Thus, $T_{1}, T_{2}, D_{1}, D_{2}, D_{2}^{\prime}$ satisfy (a), (b), and (c) (i) and (ii) of V.3 with $D^{\prime}$ replaced by $D_{2}^{\prime}$.

We repeat the process at the $i$ th stage after letting $\varepsilon_{i}$ be a positive number less than $\varepsilon_{i-1}$ and $1 / 2^{i-1}$ and then substituting $\varepsilon_{i}$ for $\varepsilon_{1}, D_{i-1}^{\prime}$ for $D, D_{i}^{\prime}$ for $D_{1}^{\prime}, E_{i}$ for $E_{1}, \Delta_{i}+E_{i-1}$ for $\Delta_{1}, D_{i}$ for $D_{1}$, and $T_{i}$ for $T_{1}$. Thus for each $i, T_{1}, T_{2}, \cdots, T_{i}$, $D_{1}, D_{2}, \cdots, D_{i}, D_{i}^{\prime}$ satisfy (a), (b), and (c) (i) and (ii) of V.3 with $D^{\prime}$ replaced by $D_{i}^{\prime}$.

By (4.7)

$$
D_{i}^{\prime}\left|\Delta_{i}+E_{i-1}+\left(\Delta-\operatorname{int} E_{i}\right)+\operatorname{Bd} \Delta=D_{i-1}\right| \Delta_{i}+E_{i-1}+\left(\Delta-\operatorname{int} E_{i}\right)+\operatorname{Bd} \Delta
$$

and, since $E_{i} \supset \Delta_{i}+E_{i-1}$ and $\left\{\operatorname{Bd} \Delta_{i}\right\}$ converges to $\operatorname{Bd} \Delta$, every $p \in \operatorname{int} \Delta$ is in $\Delta_{i}+E_{i-1}$ for some $i$ and thus $D_{j}^{\prime}(p)=D_{i-1}^{\prime}(p)$, for all $j \geqq i$. In addition, for each $i, D_{i}^{\prime}|\operatorname{Bd} \Delta=D| \operatorname{Bd} \Delta$. Also the diameter of each component of $\Delta-E_{i}$ approaches zero as $i$ approaches infinity and, for all $p$, the distance between $D_{i}(p)$ and $D_{i+1}^{\prime}(p)$ is less than $\varepsilon_{i+1}<1 / 2^{i}$. Thus $D^{\prime}=\lim D_{i}^{\prime}=\lim D_{i}$ is the $s$-disk desired for V.3.

5. Proof of V.4. Let $D^{\prime},\left\{D_{i}\right\},\left\{T_{i}\right\}$ be as given in the conclusion to V.3. Suppose $\alpha$ is a subdivision of $M-W$ so that $\left|D^{\prime}\right|-W+\sum T_{i}$ is a subcomplex of $\alpha(M-W)$.

For $i=1,2, \cdots$, Theorem III.5 [applied to $\left(M-\operatorname{int} T_{i}, \mathrm{Bd} T_{i}+J\right)$ ] (see previous footnote) shows that there is an $s$-disk $D_{i}^{\prime}$ such that $\left(D_{i}^{\prime}, \mathrm{Bd} D_{i}^{\prime}\right)$ is a conservative $\delta_{i}$-alteration of $\left(D_{i}, \operatorname{Bd} D_{i}\right)$, and $\left|D_{i}^{\prime}\right|$ is related to $\left|D_{i}\right|$ as $\left|D^{\prime}\right|$ is related to $\left|D^{*}\right|$ in the Addendum. We choose $\delta_{i}$ and $n(i)$ so that

$$
\left(\delta_{i} \text {-neighborhood of } S\left(D_{i}^{\prime}\right)\right) \subset \operatorname{st}\left[S\left(D_{i}^{\prime}\right), \alpha^{n(i)}\left(M-\operatorname{int} T_{i}\right)\right] \subset M-J .
$$

Thus, since $S\left(D_{i}^{\prime}\right)$ contains, if anything, only crossing pinch points, $S\left(D_{i}^{\prime}\right)$ is empty because $\mid$ int $D_{i}^{\prime} \mid \subset M-\operatorname{int} T_{i}$. We also assume that each $\left|D_{i}^{\prime}\right|$ is in general position with respect to each $\mathrm{Bd} T_{j}$.

For each $i$ there is a positive integer $k(i)$ such that $\left|D_{j}\right| \supset\left|D^{\prime}\right| \cap\left(M-\operatorname{int} T_{i}\right)$ for all $j \geqq k(i)$. Let $U_{i}=\operatorname{st}\left[S\left(D_{i}^{\prime}\right), \alpha^{n(i)}\left(M-\operatorname{int} T_{i}\right)\right]$. Then, for all $i$ and for all $j \geqq k(i)$

(a) $\left(\left|D_{j}^{\prime}\right|-\left(U_{i}+\operatorname{int} T_{i}\right)\right)=\left(\left|D^{\prime}\right|-\left(U_{i}+\operatorname{int} T_{i}\right)\right)$, and

(b) $\left|D_{j}^{\prime}\right|-\operatorname{int} T_{i}$ is related to $\left|D^{\prime}\right|-$ int $T_{i}$ as $\left|D^{\prime}\right|$ is related to $\left|D^{*}\right|$ in the Addendum. 
There are only finitely many ways of putting things in $U_{i}$ so that the Addendum is satisfied. Thus for some strictly increasing sequence of positive integers $\{n(1, i)\}$, $n(1,1) \geqq k(1)$, the pairs $\left[U_{1},\left|D_{n(1, i)}\right| \cap U_{1}\right]$ are all pwl homeomorphic for $i=1,2,3, \cdots$. Likewise there is a subsequence of $\{n(1, i)\}$ which we call $\{n(2, i)\}$ such that $n(2, i) \geqq k(n(1,1))$ and, for $i=1,2,3, \cdots$, the pairs

$$
\left[U_{n(1,1)}\left|D_{n(2, i)}^{\prime}\right| \cap U_{n(1,1)}\right]
$$

are all pwl homeomorphic. In this way we get a sequence of sequences $\{n(1, i)\}$, $\{n(2, i)\},\{n(3, i)\}, \cdots$ such that $\{n(j, i)\}_{i=1}^{\infty}$ is a subsequence of $\{n(k, i)\}_{i=1}^{\infty}$ for all $k<j$, and, for each fixed $k$, the pairs $\left[U_{n(k, 1)},\left|D_{n(k+1, i)}^{\prime}\right| \cap U_{n(k, 1)}\right]$ are pwl homeomorphic for $i=1,2, \cdots$.

Set $m(i)=n(i, 1)$, for $i=1,2, \cdots$. By moving things slightly in $\Sigma U_{m(i)}$ we can suppose that

$$
\left|D_{m(i)}^{\prime}\right|-\operatorname{int} T_{m(j)}=\left|D_{m(k)}^{\prime}\right|-\operatorname{int} T_{m(j)} \text {, for all } i, k>j .
$$

The (nonsingular) $s$-disks $D_{m(i)}^{\prime}$ are not nice enough because their limit might not be a disk. However, we shall choose certain subdisks and alter them to produce a nonsingular disk with boundary $J$.

Let $E_{1}$ be a sub-s-disk of $D_{m(1)}^{\prime}$ such that

$$
J+\mathrm{Bd} T_{m(1)} \text { contains }\left|\mathrm{Bd} E_{1}\right| .
$$

Let $E_{2}$ be a sub-s-disk of $D_{m(2)}^{\prime}$ such that

$$
J \cap\left|E_{2}\right| \subset\left|\mathrm{Bd} E_{2}\right| \subset J+\mathrm{Bd} T_{m(1)} \text { and }\left|\mathrm{Bd} E_{1}\right| \subset\left|E_{2}\right| .
$$

By induction, pick $E_{n}$ to be a sub-s-disk of $D_{m(n)}^{\prime}$ such that

$$
J \cap\left|E_{n}\right| \subset\left|\operatorname{Bd} E_{n}\right| \subset J+\operatorname{Bd} T_{m(n-1)} \text { and }\left|\operatorname{Bd} E_{n-1}\right| \subset\left|E_{n}\right| .
$$

Proposition V.7. $J \subset \liminf \left\{\left|E_{i}\right|\right\}$.

Proof. By (5.2), $J \cap\left|E_{i}\right| \subset J \cap\left|E_{i+1}\right|$. Therefore, we need only show that every point of $J-W$ belongs to some $\left|E_{i}\right|$. Let $q$ be any point of $J \cap\left|E_{1}\right|$ and let $p \in J-W$. For some positive integer $r, p \in M-T_{m(r)}$. Now suppose that $p \notin\left|E_{r+j}\right|$, for every $j \geqq 1$. Then, for each $j \geqq 1, T_{m(r+j-1)} \cap D_{m(r+j)}^{\prime}$ separates $p$ from $q$ in $D_{m(r+j)}^{\prime}$ and, because a disk is unicoherent, one component of $T_{m(r+j-1)} \cap D_{m(r+j)}^{\prime}$ separates $p$ from $q$. But $(p+q) \notin T_{m(r+j-1)}$ and each component of $T_{m(r+j-1)}$ has diameter less than $1 / 2^{m(r+j-1)}$. We conclude that, for every $\varepsilon$, there is a subset $R$ of $J$ which is of diameter less than $\varepsilon$ and which is within $\varepsilon$ of $W$, such that $R$ separates $p$ from $q$. But, since neither $p$ nor $q$ belong to $W$, some point of $W$ must separate $p$ from $q$ in $J$. This is a contradiction since no scc is separated by a single point. This proves V.7.

Proposition V.8. For every positive integer $r$, there is a positive integer $s(r)$, such that, for all $i, j \geqq s(r)$, 


$$
\left|E_{j}\right|-\operatorname{int} T_{m(r)}=\left|E_{i}\right|-\operatorname{int} T_{m(r)}
$$

Proof. $D_{m(r+1)}^{\prime}-\operatorname{int} T_{m(r)}$ has finitely many components and if, for some $i,\left|E_{i}\right|$ intersects one of these components, then it contains the whole component. For each component $C$ of $\left(D_{m(r+1)}^{\prime}-\operatorname{int} T_{m(r)}\right)$, let $n(C)$ be the least integer such that $C \subset\left|E_{n(C)}\right|$ and set $n(C)=0$ if $C$ intersects no $\left|E_{i}\right|$. The $s$ desired by V.8 is the maximum of the $n(C)$ 's over all components $C$ of $D_{m(r+1)}^{\prime}-\operatorname{int} T_{m(r)}$.

Define $s^{n}(r)=s\left(s^{n-1}(r)\right)$.

We now change the $E_{i}$ 's into an expanding sequence of disks in a countable number of steps.

Step 1. Let $F_{1}$ be the singular $s$-disk gotten by removing from $E_{s(1)}$ the interior of $\mathrm{Bd} E_{1}$ in $E_{s(1)}$ (see (5.2)) and replacing it by $E_{1}$. Formally, let $\Delta^{\prime}$ be the subdisk of $\Delta$ bounded by $E_{s(1)}^{-1}\left(\operatorname{Bd} E_{1}\right)$; and let $f$ be a homeomorphism of $\Delta$ onto $\Delta^{\prime}$ such that

$$
\left(E_{s(1)} \mid \operatorname{Bd} \Delta^{\prime}\right) \circ(f \mid \operatorname{Bd} \Delta)=E_{1} \mid \operatorname{Bd} \Delta .
$$

Then $F_{1}$ equals $E_{s(1)}$ on $\Delta-$ int $\Delta^{\prime}$ and $E_{1} \circ f^{-1}$ on $\Delta^{\prime}$. The singularities $S\left(F_{1}\right)$ are contained in $M-T_{m(1)}$. Let $\delta_{1}=\frac{1}{2}$ (distance from $S\left(F_{1}\right)$ to $\left.T_{m(1)}\right)$ and apply IV.3 of [4] to get a nonsingular $s$-disk $F_{1}^{\prime}$ which is a conservative $\delta_{1}$-alteration of $F_{1}$ such that $\operatorname{Bd} F_{1}^{\prime}=\operatorname{Bd} F_{1}=\operatorname{Bd} E_{s(1)}$. Note that $F_{1}^{\prime} \subset M-T_{m(s(1))}^{\prime}$.

Step 2. Let $F_{2}$ be the singular disk gotten by removing from $E_{s^{2}(1)}$ the interior of $\mathrm{Bd} E_{s(1)}$ in $E_{s^{2}(1)}$ and replacing it by $F_{1}^{\prime}$. Since

$$
\left|E_{s(1)}\right|-\operatorname{int} T_{m(1)}=\left|E_{s^{2}(1)}\right|-\operatorname{int} T_{m(1)}, \quad\left|F_{2}\right|-\left|F_{1}^{\prime}\right| \subset \operatorname{int} T_{m(1)} .
$$

Thus, because

$$
F_{1}^{\prime} \subset M-T_{m(s(1))}, \quad S\left(F_{2}\right) \subset \operatorname{int} T_{m(1)}-T_{m(s(1))} .
$$

Let $\delta_{2}=\frac{1}{2}$ (distance from $S\left(F_{2}\right)$ to $\left.T_{m(s(1))}\right)$ and apply IV.3 of [4] to get a nonsingular $s$-disk $F_{2}^{\prime}$ which is a conservative $\delta_{2}$-alteration of $F_{2} . F_{2}^{\prime}$ has the following properties:

$$
\begin{gathered}
E_{1} \text { is a sub-s-disk of } F_{2}^{\prime} . \\
\operatorname{Bd} F_{2}^{\prime}=\operatorname{Bd} F_{2}=\operatorname{Bd} E_{s^{2}(1)} . \\
F_{2}^{\prime} \subset M-T_{m\left(s^{2}(1)\right)} \\
F_{1}^{\prime}-T_{m(1)}=F_{2}^{\prime}-T_{m(1)} .
\end{gathered}
$$

Step $n(n=3,4, \cdots)$. Let $F_{n}$ be the singular $s$-disk gotten by removing from $E_{s^{n}(1)}$ the interior of $\mathrm{Bd} E_{s^{n-1}(1)}$ in $E_{s^{n}(1)}$ and replacing it by $F_{n-1}^{\prime}$ (see (5.2) and $\left.(5.4)_{n-1}\right)$. By V.8, $\left|F_{n}\right|-\left|F_{n-1}\right| \subset \operatorname{int} T_{m\left(s^{n-2}(1)\right)}$. Thus, by $(5.5)_{n-1}$,

$$
S\left(F_{n}\right) \subset \operatorname{int} T_{m\left(s^{n-2}(1)\right)}-T_{m\left(s^{n-1}(1)\right)} \text {. }
$$


Let $\delta_{n}=\frac{1}{2}$ (distance from $S\left(F_{n}\right)$ to $\left.T_{m\left(s^{n-1}(1)\right)}\right)$ and apply IV.3 of [4] to get a nonsingular $s$-disk $F^{\prime}$ which is a conservative $\delta_{n}$-alteration of $F_{n} . F_{n}^{\prime}$ has the following properties.

$$
\begin{gathered}
F_{n-2}^{\prime} \text { is a sub-s-disk of } F_{n}^{\prime} . \\
\operatorname{Bd} F^{\prime}=\operatorname{Bd} E_{s^{n}(1)} . \\
F_{n}^{\prime} \subset M-T_{m\left(s^{n}(1)\right)} . \\
F_{n-1}^{\prime}-T_{m\left(s^{n-1}(1)\right)}=F_{n}^{\prime}-T_{m\left(s^{n-1}(1)\right)} .
\end{gathered}
$$

Define $E_{1} \equiv F_{0}^{\prime}$.

We now use the $F_{i}^{\prime}$ 's to construct a nonsingular $s$-disk $D$ whose boundary is $J$.

Proposition V.9. For all $m \geqq n \geqq 2$ and for all onto homeomorphisms $g: \Delta \rightarrow \rightarrow \Delta$, there is an onto homeomorphism $h_{n}^{m}(g): \Delta \rightarrow \rightarrow \Delta$, such that

$$
\left(F_{m}^{\prime} \circ h_{n}^{m}(g)\right)\left|\left(F_{n}^{\prime} \circ g\right)^{-1}\right| F_{n-2}^{\prime}\left|=\left(F_{n}^{\prime} \circ g\right)\right|\left(F_{n}^{\prime} \circ g\right)^{-1}\left|F_{n-2}^{\prime}\right| \text {. }
$$

Proof. There is essentially only one way of extending a disk $\Delta^{\prime}$ to a larger disk $\Delta$ when $\Delta^{\prime} \cap \mathrm{Bd} \Delta$ is given. [That is to say, given $\Delta^{\prime} \subset \Delta_{1}$ and $\Delta^{\prime} \subset \Delta_{2}$ such that $\Delta^{\prime} \cap \operatorname{Bd} \Delta_{1}=\Delta^{\prime} \cap \operatorname{Bd} \Delta_{2}$, there is a homeomorphism of $\Delta_{1}$ onto $\Delta_{2}$ fixed on $\Delta^{\prime}$.] From (5.2) and (5.4) we conclude that, for $m \geqq n, F_{m}^{\prime-1}\left(\left|\operatorname{Bd} F_{n-2}^{\prime}\right|\right) \cap \operatorname{Bd} \Delta$ $=F_{m}^{\prime-1}\left(\left|\mathrm{Bd} F_{n-2}^{\prime}\right| \cap J\right)$. Proposition V.9 now follows.

Using V.9, define

$$
\begin{aligned}
& F_{0}^{\prime \prime} \equiv F_{0}^{\prime}=E_{1}, \\
& F_{1}^{\prime \prime} \equiv F_{1}^{\prime}, \\
& F_{2}^{\prime \prime} \equiv F_{2}^{\prime},
\end{aligned}
$$

and, for $n=3,4,5, \cdots$,

$$
F_{n}^{\prime \prime}=F_{n}^{\prime} \circ h_{n-1}^{n}\left(F_{n-1}^{\prime-1} \circ F_{n-1}^{\prime \prime}\right) \text {. }
$$

The reader can check that $F_{n}^{\prime \prime}, n=2,3, \cdots$, satisfies $(5.3)_{n}-(5.6)_{n}$ with all primes (') replaced by double-primes ("). In addition, if we define $\Delta_{i}=F_{i+2}^{\prime \prime-1}\left(\left|F_{i}^{\prime \prime}\right|\right)$,

$$
\text { for all } m \geqq n+2 \geqq 4, \quad F_{m}^{\prime \prime}\left|\Delta_{n}=F_{n+2}^{\prime \prime}\right| \Delta_{n} \text {. }
$$

This follows from V.9.

Define $D\left|\Delta_{i} \equiv F_{i+2}^{\prime \prime}\right| \Delta_{i}$. By (5.7), $D$ is a $1-1$, continuous map of $\sum_{i=1}^{\infty} \Delta_{i}$ into $M$. Since each component of $T_{i}$ is of diameter less than $1 / 2^{i},(5.5)$ and (5.6) show that $D$ can be extended to a $1-1$, continuous map (and thus, an embedding) of $\Delta$ into $M$. It follows from V.7 that $J \subset D(\Delta)$ and from (5.2) and (5.4) that $J=|\operatorname{Bd} D|$.

This completes the proof of V.6. 
6. Proof of V.5. Let $D$ be the nonsingular $s$-disk promised by Theorem V.4 and let $\varepsilon_{1}, \delta\left(\varepsilon_{1}^{\prime}\right)$, and $T_{1}$ be as in (4.1)-(4.5) with the additional requirement that $T_{1}$ be a $\gamma$-set, where $\gamma$ is less than $\frac{1}{2} \delta\left(\varepsilon_{1}\right)$ and so small that if $p$ and $q$ are points of $J$ within $\gamma$ of each other then one of the components of $J-(p+q)$ has diameter less than $\frac{1}{2} \delta\left(\varepsilon_{1}\right)$. Assume that $D$ is polyhedral $\bmod W$ and that $D$ and $\operatorname{Bd} T_{1}$ are in general position.

Let $C$ be a component of $T_{1}$ such that $\mathrm{Bd} C \cap J$ has four points. Call these four points $p_{1}, p_{2}, p_{3}$, and $p_{4} \cdot D^{-1}(\mathrm{Bd} C \cap|D|)$ is a finite collection of scc's and spanning arcs in $\Delta$. Since the only possible end points for $D^{-1}(\operatorname{Bd} C \cap|D|)$ are $D^{-1}(\operatorname{Bd} C \cap J), D^{-1}(\operatorname{Bd} C \cap|D|)$ has two spanning arcs which are situated as in Figure 1 or Figure 2. Note that $W \cap C$ is one point, which we call $w$; and

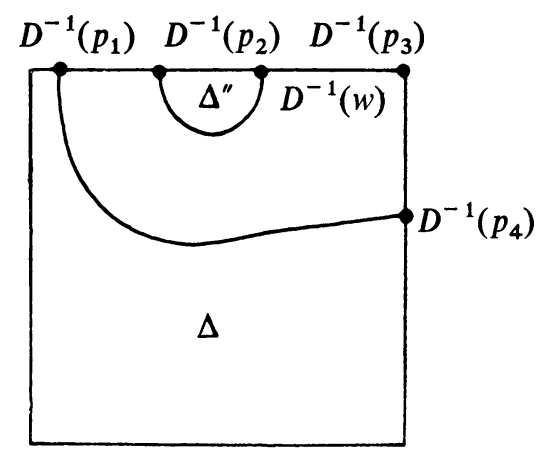

FIGURE 1

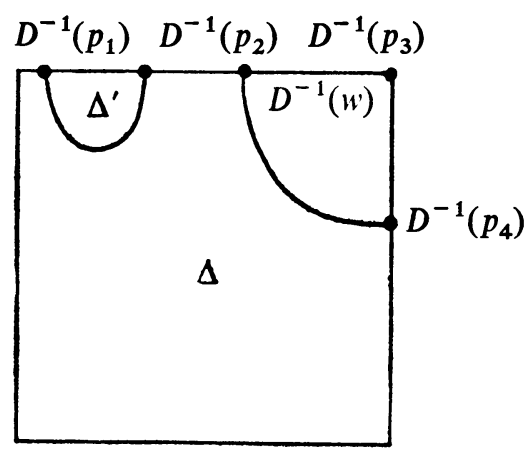

FIGURE 2

$D^{-1}(w)$ is between $D^{-1}\left(p_{1}\right)$ and $D^{-1}\left(p_{2}\right)$, or $D^{-1}\left(p_{3}\right)$ and $D^{-1}\left(p_{4}\right)$. We suppose the latter.

We shall look at the two cases depicted by Figure 1 and Figure 2.

Case depicted by Figure 1. The image of a neighborhood of $\operatorname{Bd} \Delta^{\prime \prime}$ is in $M-\operatorname{int} T_{1}$, and $\left(D \mid \Delta^{\prime \prime}\right)^{-1}\left(\operatorname{Bd} C \cap\left|\left(D \mid \Delta^{\prime \prime}\right)\right|\right)$ is a finite collection of scc's. By 
using arguments almost identical to those employed in the pro of of V.3, we can remove the scc's that bound disks on $\mathrm{Bd} C-J$ and then show that no more are left. Thus we suppose that $D$ has been altered so that

$$
D \mid \Delta^{\prime \prime} \text { is nonsingular, } \operatorname{int}\left(D \mid \Delta^{\prime \prime}\right) \subset M-T_{i} \text {, and }
$$

$$
\operatorname{Bd}\left(D \mid \Delta^{\prime \prime}\right) \subset \operatorname{Bd} C+\left(\text { small part of } J \text { between } p_{2} \text { and } p_{3}\right) \text {. }
$$

By our choice of $\gamma$, the diameter of $\left|\operatorname{Bd}\left(D \mid \Delta^{\prime \prime}\right)\right|$ is less than $\delta\left(\varepsilon_{1}\right)$ and thus the diameter of $\left|\left(D \mid \Delta^{\prime \prime}\right)\right|$ is less than $\varepsilon_{1} / 3$. (See (4.2).) By thickening up $\left|\left(D \mid \Delta^{\prime \prime}\right)\right|$ we obtain a new taming $\varepsilon_{1}$-set $T^{\prime}$ of $J$ of index $\leqq 4$ and such that $J \cap B d T^{\prime}$ has two fewer points than $J \cap B d T_{1}$. This contradicts (4.1) and (4.5).

Case depicted by Figure 2. By repetition of previous arguments we can alter $D$ on int $\Delta^{\prime}$ to remove the components of intersection with $\mathrm{Bd} C$, and thus obtain a nonsingular $s$-disk $D^{\prime}$, such that $\mid$ int $D^{\prime} \mid \subset$ int $C$ and $\mathrm{Bd} D^{\prime} \subset \mathrm{Bd} C+$ (component of $J \cap C$ between $p_{1}$ and $p_{2}$ ). By splitting $C$ apart along $\left|D^{\prime}\right|$ we can obtain a new taming $\delta\left(\varepsilon_{1}\right)$-set $T^{\prime}$ of $J$ of index $\leqq 4$ and such that $J \cap \mathrm{Bd} T^{\prime}$ has two fewer points than $J \cap B d T_{1}$. This again is a contradiction.

Thus V.5 is proven.

\title{
REFERENCES
}

1. B. J. Ball, Penetration indices and applications, Topology of 3-manifolds and related topics, Prentice-Hall, Englewood Cliffs, 1962.

2. R. H. Bing, Approximating surfaces with polyhedral ones, Ann. of Math. 61 (1957), 456-483.

3. O. G. Harrold, H. C. Griffith, and E. E. Posey, A characterization of tame curves in 3-space, Trans. Amer. Math. Soc. 79 (1955), 12-35.

4. D. W. Henderson, Extensions of Dehn's Lemma and the Loop Theorem, Trans. Amer. Math. Soc. 120 (1965), 448-469.

\author{
UNIVERSITY OF WISCONSIN, \\ MADISON, WISCONSIN \\ INSTITUTE FOR ADVANCED STUdY, \\ Princeton, New Jersey
}

